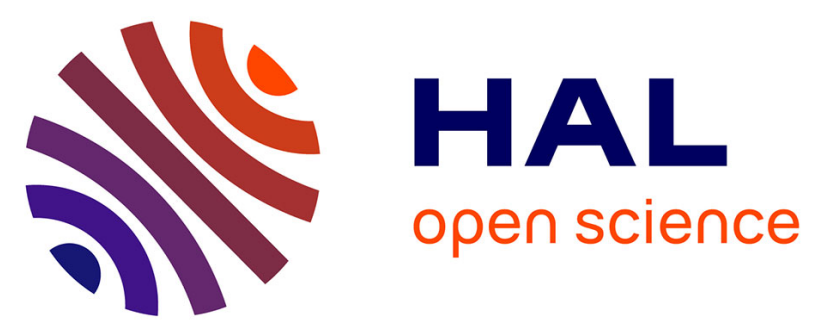

\title{
Remote Control of SAW Resonators Using a Frequency-Modulation-Based Interrogation Strategy
}

\author{
C. Droit, J.-M. Friedt, G. Martin, S. Ballandras
}

\section{To cite this version:}

C. Droit, J.-M. Friedt, G. Martin, S. Ballandras. Remote Control of SAW Resonators Using a Frequency-Modulation-Based Interrogation Strategy. 2010 IEEE International Frequency Control Symposium, Jun 2010, New Port Beach, United States. pp.503-510, 10.1109/FREQ.2010.5556277 . hal-00589216

\section{HAL Id: hal-00589216 https://hal.science/hal-00589216}

Submitted on 14 May 2021

HAL is a multi-disciplinary open access archive for the deposit and dissemination of scientific research documents, whether they are published or not. The documents may come from teaching and research institutions in France or abroad, or from public or private research centers.
L'archive ouverte pluridisciplinaire HAL, est destinée au dépôt et à la diffusion de documents scientifiques de niveau recherche, publiés ou non, émanant des établissements d'enseignement et de recherche français ou étrangers, des laboratoires publics ou privés. 


\section{Remote Control of SAW Resonators Using a Frequency-Modulation-Based Interrogation Strategy}

\author{
C. Droit, J.-M Friedt \\ SENSeOR SAS, Besançon, France \\ Email: jmfriedtefemto-st.fr
}

\author{
G. Martin, S. Ballandras \\ FEMTO-ST \\ UMR 6174 CNRS/UFC/ENSMM/UTBM \\ Besançon, France \\ Email: ballandrefemto-st.fr
}

Within the context of probing acoustic wave resonators acting as wireless, passive sensors, we present a strategy for wireless monitoring of surface acooustic wave (SAW) resonators using a frequency modulation based interrogation method. The basic principle is the conversion of frequency modulation to amplitude modulation by the resonator through its $S_{11}$ transfer function.

An ARM-7 based central processing unit synchronizes the narrow-band probing pulse emission, emission to reception switching, data sampling and data processing. A closed loop control keeps the emitted carrier frequency at the resonance frequency of the sensor: we demonstrate a resolution of $2 \mathrm{~Hz}$ on the resonance freqeuncy identification at $1 \mathrm{~s}$ integration time, limited by this proportional feedback loop.

\section{INTRODUCTION}

Surface acoustic wave devices have been demonstrated as superior passive wireless sensors with respect to passive silicon-based sensors (RFID) and with unique capabilities with respect to active silicon-based sensors, including longer lifetime thanks to the lack of local power supply and wider temperature range [1], [2]. Amongst the two classes of acoustic sensors - delay lines and resonators - we focus on the latter since the narrow-band resonator is the only strategy compatible with the European $434 \mathrm{MHz}$ ISM (Instrument-Scientific-Medical) band regulation [3]-[7]. Indeed, the $1.74 \mathrm{MHz}$ wide frequency range cannot provide the needed bandwidth for interrogating wideband delay lines. The lower ISM frequency range, with respect to the $2450 \mathrm{MHz}$ band, has been selected for manufacturing reproducibility, and improved penetration depth of the electromagnetic wave in dielectric media. The drawback of the $434 \mathrm{MHz}$ band is the large - typically $35 \mathrm{~cm}$ long - antenna associated with the sensor.

In order to probe these passive acoustic resonators, we are developing the associated radiofrequency (RF) interrogation unit (Fig. 1). The basic principle of this electronics is based on a pulse-mode RADAR strategy with alternating emission and reception steps - with unique improvements such as emitting a probe pulse with a spectral width narrower than the resonator width at half height. The aim of an interrogation by frequency modulation 
is to improve the accuracy of the resonance frequency estimate in a narrow frequency range complying with the 1.74 MHz wide $434 \mathrm{MHz}$ European ISM band. In a first time, we use a frequency-sweep network analyzer strategy to roughly (within one third of the width at half height of the resonator) identify the resonant frequency of the sensor and thus initialize the feedback loop. We will demonstrate that due to the long response time of the high qualityfactor resonator (decay time $Q / \pi$ with $Q$ the quality factor of the sensor), the frequency modulation strategy induces an increased global interrogation duration with respect to the network analyzer strategy, due to the many frequencies sampled to realize a digital filter to measure the amplitude and phase of the received amplitude-modulated signal.

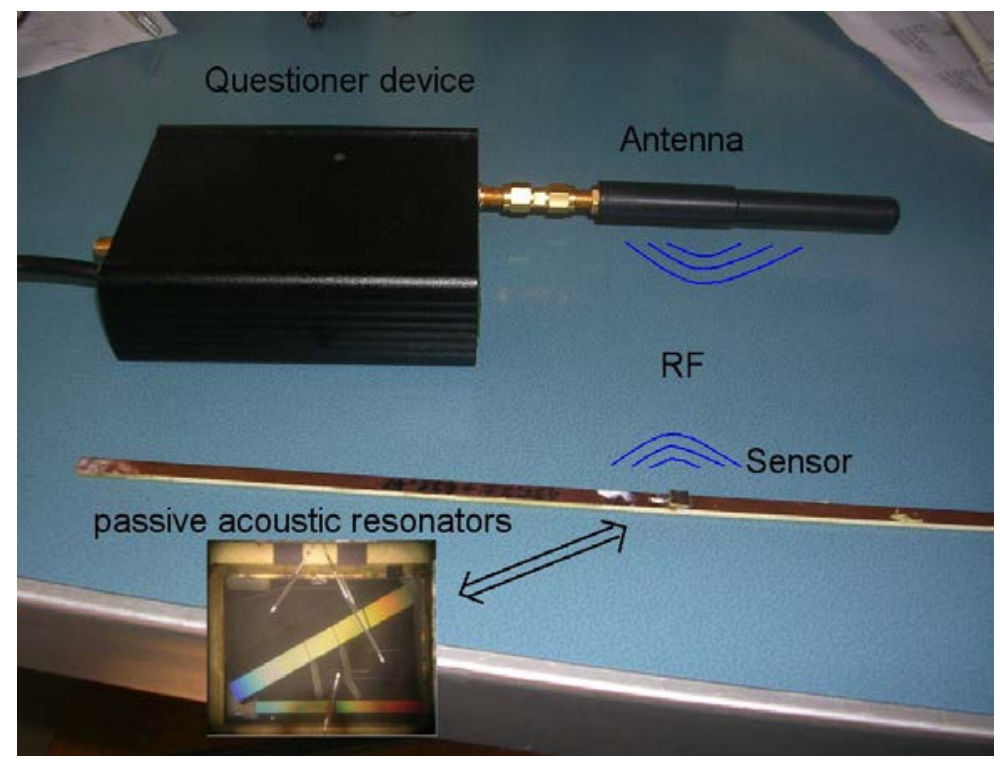

Fig. 1. Wireless sensor, associated antenna and interrogation unit.

\section{HARDWARE ARCHITECTURE}

The core component of the interrogation unit is an ARM7-based microcontroller in charge of synchronizing all the measurement steps, storing and processing the resulting data, and communicating with the user through an asynchronous serial port (Fig. 2). An Analog Devices AD9954 Direct Digital Synthesizer (DDS), programmed through a fast, local SPI bus, clocked by an intenally PLL generated $200 \mathrm{MHz}$ signal (10 times multiplication of a $20 \mathrm{MHz}$ quartz reference), generates a signal in the $34 \pm 0.85 \mathrm{MHz}$ range when complying with ISM regulations, although the full range is $0-133 \mathrm{MHz}$, providing the needed flexibility to probe sensors performing out of the ISM band. This RF signal is mixed and band-pass filtered with a $400 \mathrm{MHz}$ fixed frequency oscillator in order to generate the emitted carrier frequency. A duplexer switches between the emission amplification circuit and the receiver circuit. Switching under microcontroler control to the receiving stage once the resonator is loaded, the received signal is band-pass filtered, amplified and fed to a wideband RF-power detector for digitization following an offset removal step and low-frequency amplification. Hence, in a basic network analyzer mode, for each emitted 
frequency at varying carrier frequency, a single magnitude response is recorded, representative of the amount of energy stored in the resonator. ( [8]).

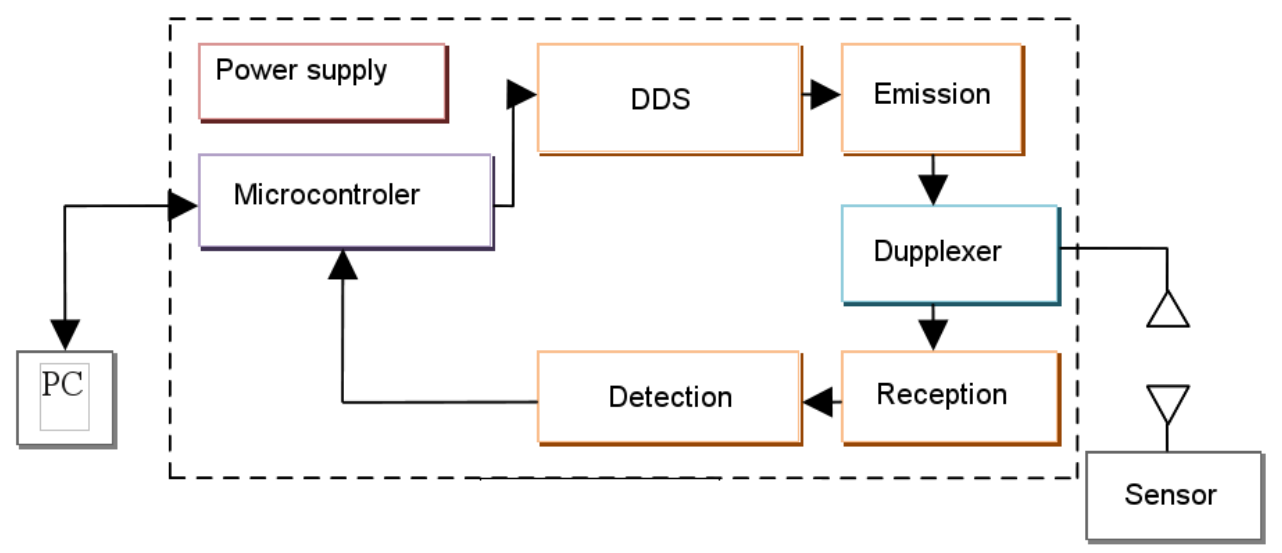

Fig. 2. Synoptic of the hardware architecture

\section{Principle of Frequency-Modulation-BASEd InterRogation Strategy}

The transfer function of a resonator acts as a frequency-to-amplitude modulation converter (Fig 3). Hence, by exciting a narrowband resonator away from its resonance frequency with a frequency modulated (FM) signal at a modulation rate $\omega_{m}$ on one of the resonance slopes, the returned signal is amplitude modulated (AM) at $\omega_{m}$. At resonance frequency, where the first order coefficient for a polynomial development of the transfer function becomes null and the second order coefficient becomes dominant, the FM at $\omega_{m}$ becomes an AM at $2 \times \omega_{m}$, with a contribution at $\omega_{m}$ close to zero. Above the resonance frequency, the FM at $\omega_{m}$ again becomes an AM at $\omega_{m}$, but this time with a 180 phase shift of the AM with respect to the FM signal.

\section{FM SYNTHESIS USING THE DDS}

The flexibility of the full software configuration of the generated frequency using the hardware designed described in Fig. 2 is demonstrated by the following description of the implementation of the FM strategy with no need to modify the board. Hence, the ability to switch through a software configuration update allows to adapt the interrogation strategy to the environment of the sensor.

FM generation is performed through the software configuration of the AD9954 DDS. Indeed, this DDS provides an internal RAM which is automatically swept at a programmable rate: the output frequency value is fetched periodically and generated. Hence, by filling the RAM with a sine-shaped set of values, a software configurable FM generator is obtained, with the FM carrier and excursion defined by the magnitude of the values stored in the RAM, and the FM frequency $\omega_{m}$ defined by the sweep rate of the RAM.

We use 100 RAM 32 bits registers filled with a sine table describing regularly spaced phases ranging from 0 to $360^{\circ}$ (Fig 4). The DDS has different programming modes, one of which is called "RAM UP": in this mode, the 


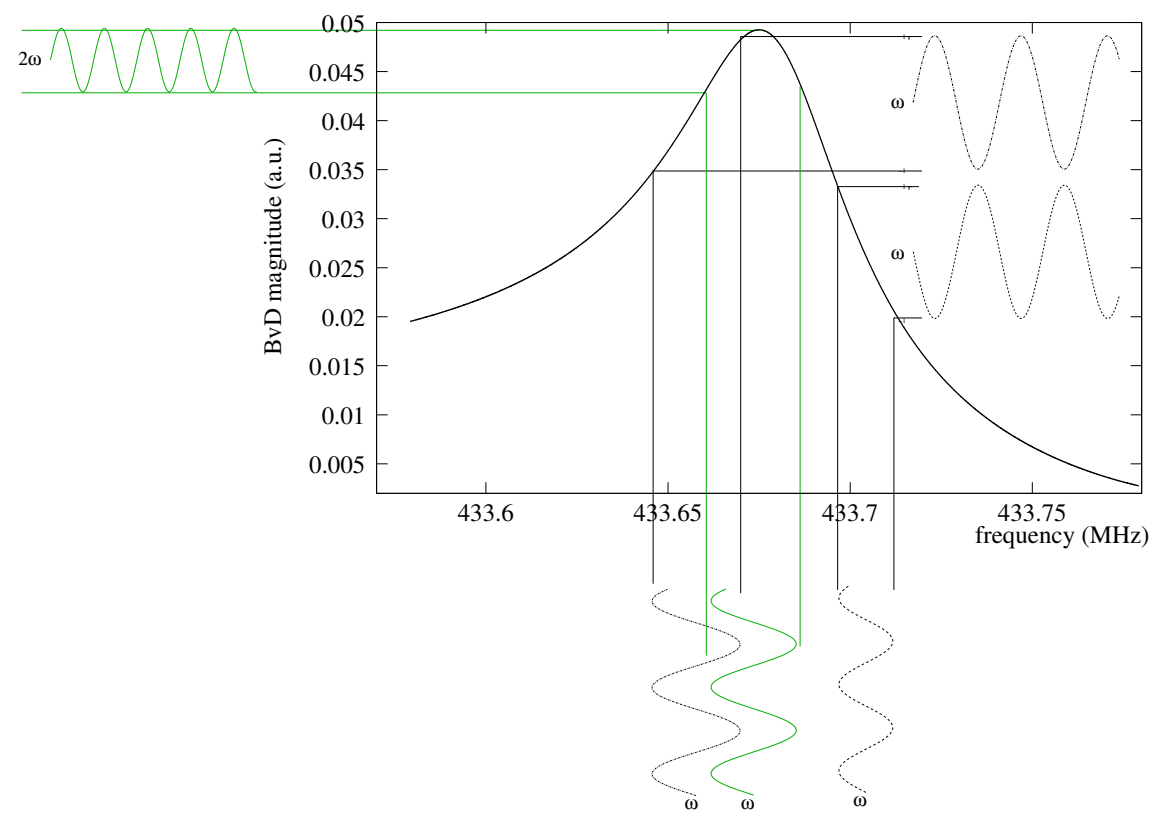

Fig. 3. Frequency to amplitude modulation through the transfer function of the resonator: at resonance, the contribution at modulation pulsation $\omega_{m}$ vanishes and the sole contribution to the returned signal is at multiples of $2 \times \omega_{m}$. From left to right: When the slope of the transfer function is rising, the emitted FM signal is recovered as an AM signal. At resonance frequency, the contribution at $\omega_{m}$ vanishes. When the slope of the transfer function is negative, the emitted signal at modulation frequency $\omega_{m}$ is received as an AM signal at $\omega_{m}$ but with phase opposition.

DDS scans the RAM with a user-defined periodicity. The carrier is a fixed value for a given measurement sequence, the 100-values array describing the FM are added to the carrier value and stored in the DDS RAM. Depending on the returned signal from the resonator as will be described in the following section, the carrier frequency value is updated, and a new set of values are computed by adding the 100-FM dataset and sent to the DDS when starting a new probing sequence of the resonator.

The emitted FM is defined as a function of time $t$ by $y(t)=\cos \left[\omega_{c} \times t+B \times \sin \left(\omega_{m} \times t\right)\right]$ where $B$ is the modulation excursion, $y(t)$ the output signal, $\omega_{m}$ is the modulation frequency, and $\omega_{c}$ is the carrier frequency. The numerical values used comply with the following rules:

1) European ISM regulation require that the radiofrequency signal is restricted to the $[433.05-434.79] \mathrm{MHz}$ range. Hence, $\omega_{c}$ is restricted to a subset of this interval,

2) the FM signal must probe a significant fraction of the resonance of the resonator, without significantly departing from the localy linear relationship between output magnitude and input frequency on the slopes of the transfer function. Hence, $B$ will be restricted to a range of a few $\mathrm{kHz}$ to a value lower than the width at half height of the resonance. This value is dependent on the resonator quality factor $Q$,

3 ) in pulse mode RADAR, the resonator is probed by each pulse and reacts with a time constant of $Q / \pi$. Each probe pulse must appear at a constant frequency from the resonator point of view, in order to distinguish the 
amplitude of each sample of the returned signal. Assuming that the resonator has settled after ten times its time constant - i.e. a duration $\tau_{m}=10 \times Q /\left(\omega_{c} \times \pi\right)$ - then the FM frequency cannot rise above $1 / \tau_{m}$. This limitation of $\omega_{m} \leq 1 / \tau_{m}$ will be the cause of a long interrogation duration, as will be explained in the next section.

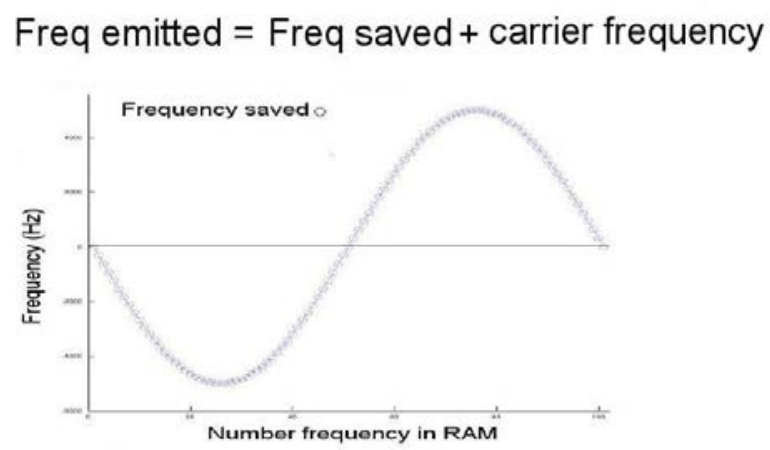

Fig. 4. Frequency saved to the RAM to create the modulation frequency

\section{FM TO AM CONVERSION INTERROGATION OF THE RESONATOR}

The resonators we use exhibit $Q \geq 10000$ around $\omega_{c} \sim 434 \mathrm{MHz}$. Hence $\tau_{m}=7.3 \mu$ s and $\omega_{m} \leq 13 \mathrm{kHz}$. As we use a pulse-mode RADAR strategy, each interrogation step requires distinct emission and reception steps, with the former loading the resonator with energy and the latter used to record the returned signal (analog to digital conversion), store the result in memory, compute the next measurement parameters and wait for the resonator to fully unload (Fig 5): both tasks last equal durations for a total measurement time of $60 \mu s$ for each recorded response sample. Hence, we replace the value of $\tau_{m}$ previously computed with $\tau_{m}=60 \mu$ s, and hence $\omega_{m} \leq 1700 H z$ : the RAM address increment rate to generate the FM is limited by the charge and discharge durations of the resonator. In practice, we tune the measurement parameters to reach $\omega_{m}=2 \mathrm{kHz}$, and the $100 \mathrm{RAM}$ registers are swept at a $200 \mathrm{kHz}$ rate.

The resonance frequency identification requires a phase detection strategy: in order to improve the signal to noise ratio of the returned AM amplitude and phase with respect to the emitted FM signal, we record 5 periods. The resulting dataset is digitally band-pass filtered using a 80-coefficients Finite Impulse Response (FIR) filter (Fig 6) to focus the phase and amplitude detection around $\omega_{m}$, running on 500 points (5 periods sampled with 100 steps each). We have observed that the resturned signal at $2 \omega_{m}$ is too weak to be usable in a closed loop carrier frequency tracking strategy.

The received signal is filtered at $\omega_{m}$ (Fig 6). We could interrogate the resonator with a frequency more high, but we are limited too by the sample frequency. In a first time, the acquisition of signal is managed by a periodical time controlled by interruption timer. The probleme is if we use the timer of microcontroller, we are limited to 16 $\mathrm{kHz}$, but if we just use a software loop, we can reach $26 \mathrm{Khz}$ of sample frequency. 


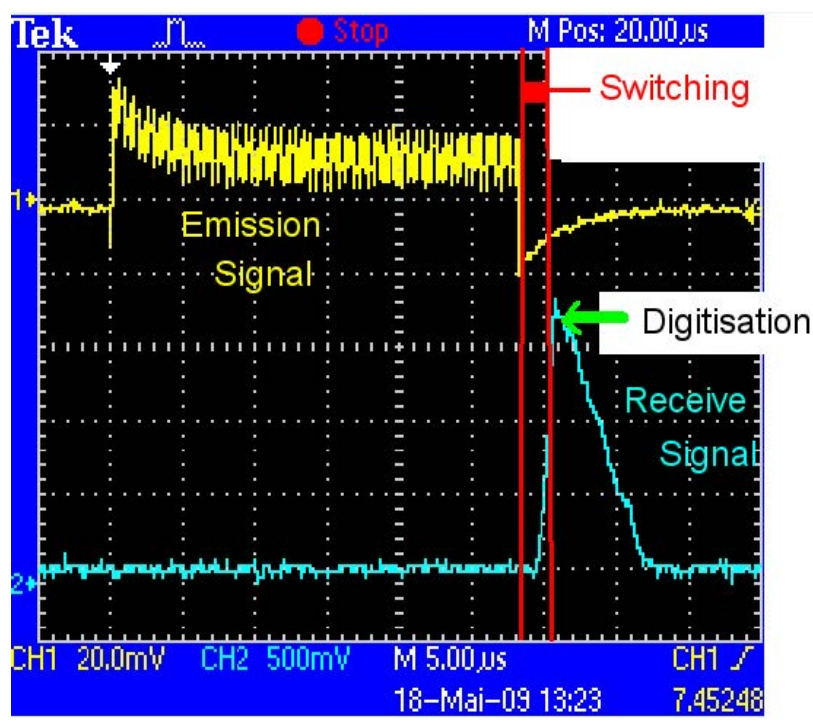

Fig. 5. Probe pulse emission (yellow curve) and received signal at the wideband power detector output (blue curve). Notice the exponential decay of the received power envelope of about $6 \mu \mathrm{s}$, yielding the need for a fast (better than $1 \mathrm{Msamples} / \mathrm{s}$ ) analog to digital converter at the power detector output.

\section{USE OF FEEDBACK LOOP}

To confirm the practical validation of the strategy we described here, we record the result of the frequencymodulated method used with $\omega_{m}=2 \mathrm{kHz}$, using the digital implementation of the the FIR filter described in Fig. 6 (Fig 7). The feedback control aims at keeping the phase information close to 0 .

\section{ViI. Measurement Resolution}

In terms of measurement resolution, the algorithm has been characterized by interrogating a temperature sensor made of dual-SAW resonators, connected to the antenna output through a $25 \mathrm{~dB}$ attenuator simulating a fixed electromagnetic environment. We observe a standard deviation of $25 \mathrm{~Hz}$ limited by the stability of the resonator. We have demonstrated sub-10 Hz stability with a resonator attached to a heat sink [9]. We compare the evolution of the standard deviation of the fixed frequency comb strategy and the FM strategy as a function of the FM excursion. The FM strategy with feedback loop exhibits a noise level of $1 \mathrm{~Hz}$ when configured with appropriate parameters, yielding sub-mK temperature resolution. However, the FM strategy is only suitable for physical quantities varying slowly because of the increased time acquisition, and is especially unsuitable with mobile sensors.

\section{CONCLUSION}

We have demonstrated the use a flexible radiofrequency generation system centered on a microprocessor-controlled direct digital synthesizer and a duplexer for switching between an emission phase and a reception phase for probing wireless passive acoustic resonators used for sensing purposes. We have demonstrated a strategy for interrogating acoustic resonators based on the cancellation of an amplitude modulated signal resulting from the conversion of the 


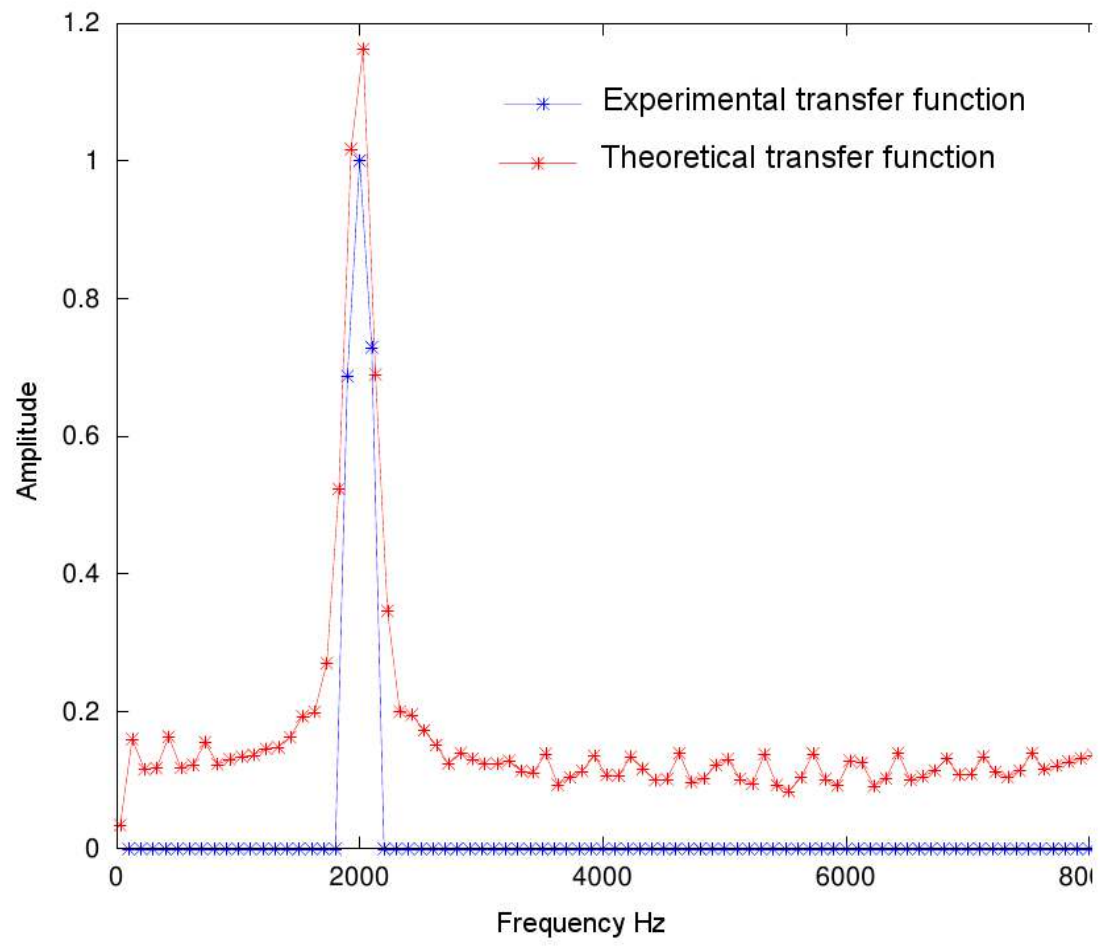

Fig. 6. Simulation of the FIR filter response, and experimental assessment of the performance of the filter applied to data recorded through the analog to digital converter of the ADuC7026 microcontroller.

FM emitted signal to an amplitude modulation through the transfer function of the resonator. With this strategy, the feedback loop control yields a sub- $25 \mathrm{~Hz}$ resolution in the indentification of the resonance frequency of the device operating in the $434 \mathrm{MHz}$ European ISM band.

\section{REFERENCES}

[1] F. Seifert, A. Pohl, R. Steindl, L. Reindl, M.J. Vellekoop, and B. Jakoby, Wirelessly interrogable acoustic sensors, Proceeding of the Joint Meeting EFTF-IEEE IFCS (1999), 1013-1018

[2] W.E. Bulst and G. Fischerauer and L. Reindl, State of the Art in Wireless Sensing with Surface Acoustic Waves, IEEE Transactions on Industrial Electronics, 48 (2) (2001), 265-271

[3] W. Buff, F. Plath, O. Schmeckebier, M. Rusko, T. Vandahl, H. Luck, F. Möller, D.C. Malocha, Remote Sensor System Using Passive SAW Sensors, IEEE Ultrasonics Symposium, (1994), 585-588

[4] U. Rösler, W. Ruile, K.Ch. Wagner, T.W. Johannes, G. Scholl, R. Weigel, Energy distribution in a Quartz resonator, IEEE Ultrasonics Symposium, (1996) 1-4

[5] A. Pohl, G. Ostermayer, F. Seifert, Wireless Sensing Using Oscillator Circuits Locked to Remote High-Q SAW Resonators, IEEE transactions on ultrasonics, ferroelectrics, and frequency control 45 (5) (1998) 1061-1168

[6] Y. Wen, P. Li, J. Yang, M. Zheng, Detecting and Evaluating the Signals of Wirelessly Interrogational Passive SAW Resonator Sensors, IEEE sensors journal 4 (6) (2004), 828-836

[7] V. Kalinin, Influence of Receiver Noise Properties on Resolution of Passive Wireless Resonant SAW Sensors, IEEE Ultrasonics Symposium, 3 (2005), 1452-1455 


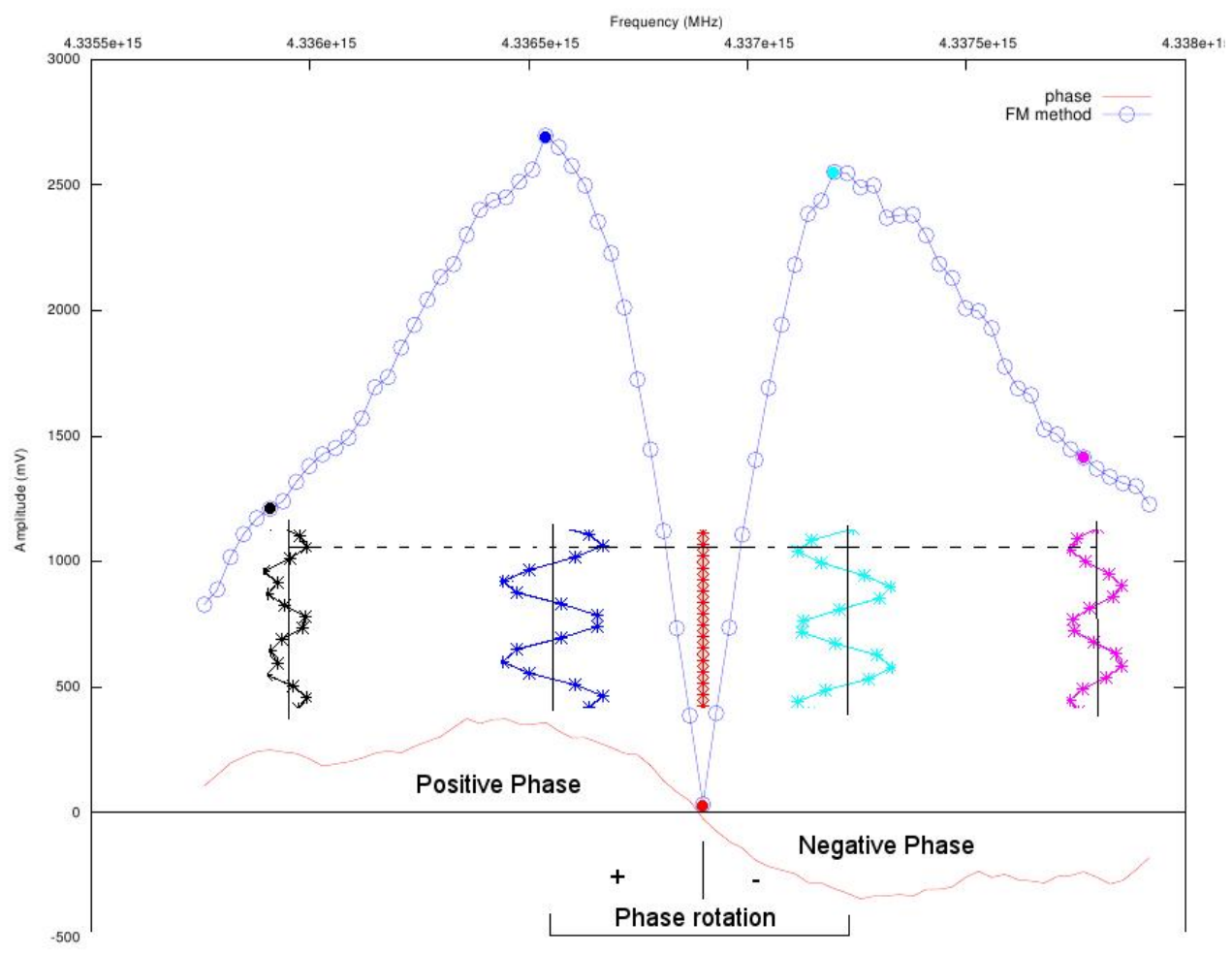

Fig. 7. Received power as a function of emitted frequency close to resonance - the modulation excursion is $B=10 \mathrm{kHz}$ and the modulation frequency is $\omega_{m}=2 \mathrm{kHz}$ - and signed magnitude (phase). The signed (red) amplitude is equal to the magnitude signal (blue) with the additional information of the phase of the received amplitude modulated signal with respect to the emitted modulation signal. The red curve is hence the so-called phase information, with a sign change and a locally linear variation near 0 , consistent with a closed loop feedback operation, as opposed to the notch observed on the magnitude signal which yields unstable feedback control.

[8] J.-M Friedt, C. Droit, G. Martin, S. Ballandras, A wireless interrogation system exploiting narrowband acoustic resonator for remote physical quantity measurement, Rev. Sci. Instrum 81 (2010), 014701

[9] C. Droit, S. Ballandras, G. Martin, J.-M Friedt A frequency modulated wireless interrogation system exploiting narrowband acoustic resonator for remote physical quantity measurement, accepted Rev. Sci. Instrum. (2010) 